\title{
Hipertiroidismo y Psicosis en Pediatría: Reporte de Caso
}

\author{
Fred Cavallo Aita ${ }^{1}$ \\ Gustavo Mendoza Alfaro. ${ }^{2}$ \\ Berny Roldán Abellán
}

\author{
${ }^{1}$ Médico Asistente Especialista en Endocrinología pediátrica. Hospital Nacional de Niños. C.C.S.S. Correo \\ electrónico: fcavalloa@gmail.com. (Autor principal) \\ ${ }^{2}$ Médico residente de Pediatría. Hospital Nacional de Niños. C.C.S.S. \\ ${ }^{3}$ Médico general. Universidad de Costa Rica. Correo electrónico: balonsora@gmail.com
}

\section{RESUMEN}

La enfermedad de Graves es un desorden autoinmune que constituye la principal causa de hipertiroidismo en niños; usualmente se manifiesta con bocio difuso, signos de oftalmopatía bilateral y la alteración bioquímica en las pruebas de función tiroidea. En ocasiones, los síntomas iniciales se presentan con manifestaciones psiquiátricas, lo que llega a ser un reto en el diagnóstico diferencial, ya sea del trastorno tiroideo primario o un trastorno psiquiátrico. Debido a la poca frecuencia con que esta manifestación de la enfermedad de graves se presenta en pediatría se expone aquí el caso de hipertiroidismo y psicosis aguda en un paciente pediátrico.

\section{PALABRAS CLAVE}

Hipertiroidismo, Niños, Enfermedad de Graves, Psicosis aguda

\section{ABSTRACT}

Graves's disease is the most common cause of hyperthyroidism in children and adolescents. Generally presents itself as a diffuse goiter, clinical findings of bilateral ophthalmopathy and abnormal laboratory results in the thyroid function test. Sometimes, the psychiatric manifestations become the initial symptoms, what turns the differential diagnoses, from either the primary
\end{abstract}

thyroid disorder or the psychiatric disease, in quite a challenge. Because of the low incidence of psychosis as manifestation of Graves' disease in children, we present a case of hyperthyroidism and acute psychosis in a child.

\section{KEYWORDS}

Hyperthyroidism, children, Graves' disease, Psychosis.

\section{CASO CLINICO}

Un paciente masculino, costarricense, de 12 años de edad, fue admitido en el Hospital nacional de niños "Dr. Carlos Sáenz Herrera" por historia de alteración del comportamiento, bocio, proptosis, y alucinaciones visuales.

El paciente es conocido sano, viviendo en albergue del PANI (Patronato Nacional de la Infancia, Costa Rica), con historia de alucinaciones que consistía en ver un "niño de 5 años de edad, pequeño, vestido de blanco, con espirales rojas en el pecho, el cual le decía que quemara el albergue donde se encontraba en ese momento, ya que no era un buen lugar". Las alucinaciones visuales del paciente durante esa semana se hicieron más frecuentes y además, comenzó a presentar un comportamiento agresivo, apático, ansioso, asociando irritación ocular, diaforesis, insomnio e inquietud por lo que los encargados del menor deciden consultar al hospital nacional psiquiátrico. Fue valorado 
inicialmente como un trastorno de comportamiento, se le realizó una evaluación psiquiátrica integral y entre los exámenes se realizaron pruebas de función tiroidea que evidenciaron TSH: $0.0 \mathrm{mUI} / \mathrm{L}$ (rango normal: 0.4$4.5 \mathrm{mU} / \mathrm{L}$ ), T3: $13.2 \mathrm{ng} / \mathrm{dL}$ (rango normal: 71-180 ng/dL) y T4 libre: $2.69 \mathrm{ng} / \mathrm{dL}$ (rango normal: 0.93-1.71 ng/dL), por lo cual fue referido al Hospital Nacional de Niños para descartar endocrinopatía.

Durante su valoración en el servicio de emergencias, se detectó que el niño tenía antecedente de consumo de marihuana y alcohol desde hace dos meses aproximadamente y además de su trastorno de comportamiento y alucinaciones visuales se documentó perdido peso en el último mes, insomnio e inquietud, encontrándose a menudo sudoroso en reposo, y con síntomas de diarrea intermitente.

En la exploración física se observa con peso $38 \mathrm{~kg}$ (percentilo 40 de curva de peso para edad), talla $149 \mathrm{~cm}$ (percentilo 50 de curva de talla para edad), IMC: 17.11 (entre percentilo 25 al 50), Fc: $114 \mathrm{lpm}$, PA: $124 / 78 \mathrm{mmHg}$, temperatura: $37^{\circ} \mathrm{C}$; luce adelgazado, afebril, eupneico, taquicárdico y con exoftalmos bilateral. Cuello cilíndrico con bocio grado 3 , de consistencia suave, uniforme, no doloroso a la palpación; con escasas adenopatías cervicales reactivas.

Con tremor pronunciado en ambas manos. Reflejos osteotendinosos braquiales, patelares y aquilianos hiperrefléxicos. Pulsos normales y simétricos y sin alteraciones patológicas en el resto del examen físico.

Se tomaron laboratorios con hemograma y bioquímica sin alteraciones, así como serologías virales que posteriormente resultaron negativas. Anticuerpos antitiroglobulina: 403,1 UI/mL $(<40$

$\mathrm{UI} / \mathrm{mL}$ ) anticuerpos antimicrosomales: 304,6 $\mathrm{pg} / \mathrm{mL}(<20 \mathrm{pg} / \mathrm{mL})$, anticuerpos anti-receptor de TSH: $29,99 \mathrm{UI} / \mathrm{L}(<1 \mathrm{UI} / \mathrm{L})$ y pruebas de función tiroidea, con TSH menor a $0.0005 \mathrm{mU} / \mathrm{L}$, T4 libre en $14.9 \mathrm{ng} / \mathrm{dL}$ y tiroglobulina $93.38 \mathrm{ng} / \mathrm{mL}$.

Se le realizó un ultrasonido de tiroides que evidencia un bocio difuso con tiroides mamelonada con hipercaptación de flujo, no focaliza lesiones, no hay nódulos; con adenopatías inflamatorias a nivel II derecho y III izquierdo, de 9-17 mm. Se le inició tratamiento con
Propiltiouracilo VO $150 \mathrm{mg}$ cada ocho horas y Propranolol VO $20 \mathrm{mg}$ cada doce horas.

Durante esta hospitalización se coordinó una valoración por psiquiatría; quienes decidieron iniciar tratamiento con haloperidol debido a las alucinaciones visuales.

Fue valorado además por oftalmología, sin evidenciarse alteraciones en el fondo de ojo, sin úlceras corneales o disfunción de músculos extraoculares.

En la tercera semana de evolución clínica, el niño mejoró significativamente su sintomatología y, posterior a una extensa valoración social, se decidió egresar con reforzamiento de la importancia de la adherencia al tratamiento, sin tratamiento antipsicótico y sin propanolol

La pruebas de función tiroidea control, a las 8 semanas, mostraron: TSH menor a $0.005 \mathrm{mU} / \mathrm{L}$, T4 libre $1.76 \mathrm{ng} / \mathrm{dL}$, tóxicos en orina negativos.

\section{DISCUSIÓN}

La enfermedad de Graves es un desorden autoinmune que constituye la principal causa de hipertiroidismo en niños; con una prevalencia del $1 \%$, con una presentación más frecuente entre la segunda y cuarta décadas de vida. ${ }^{(\mathbf{1})}$ Usualmente existe un bocio de tamaño moderado, difuso, y firme, y cerca del $50 \%$ de los pacientes tienen signos de oftalmopatía asociada; con exoftalmos, retracción parpebral, disfunción de los músculos extraoculares, afectación corneal y pérdida de visión. ${ }^{(1)}$

Interacciones entre susceptibilidad genética y factores ambientales estimulan el inicio de la formación de anticuerpos contra el receptor de TSH (TSH-R), localizado en la glándula tiroidea, tejido orbitario y cerebro. ${ }^{(\mathbf{1 , 2})}$

Los síntomas iniciales de esta enfermedad con poca frecuencia son de naturaleza psiquiátrica, lo cual lleva a mayor dificultad en el diagnóstico diferencial de hipertiroidismo y trastornos de comportamiento, ansiedad y depresión. ${ }^{(3)} \mathrm{Su}$ prevalencia se desconoce en la población pediátrica, debido a que estudios previos, los cuales se han realizado con muestras pequeñas, no la han incluido. A pesar de lo anterior se cree que los síntomas pseudo-psiquiátricos constituyen el 
primer signo de hipertiroidismo en el $2-12 \%$ de los casos. ${ }^{(3)}$

A diferencia del hipotiroidismo, en el que las manifestaciones psiquiátricas más frecuentes son depresión, psicosis y disfunción cognitiva; se ha demostrado que los pacientes con hipertiroidismo tienen más probabilidades de sufrir síntomas de ansiedad, psicosis, agresión, así como disfunción cognitiva, que los sujetos normales. En otros estudios también se ha observado un deterioro en las pruebas de concentración y atención realizadas por pacientes hipertiroideos comparado con personas no hipertiroideas. ${ }^{(\mathbf{1 , 3})}$

En un estudio por Robert Stern y colegas, basado en la realización de cuestionarios sobre problemas neuropsiquiátricos y de comportamiento, en 137 pacientes de la Fundación nacional de enfermedad de Graves, 78.1\% aquejaron irritabilidad, 72.3\% refirieron ansiedad, 53\% enojo y 55\% sensación de ganas de llorar o tristeza. Además, 66.4\% refirieron sufrir de insomnio, $65 \%$ fatiga y $63 \%$ pérdida de peso. Sólo $7.7 \%$ reportaron haber recibido tratamiento con medicamentos psicotrópicos previo al inicio de los síntomas de hipertiroidismo, y $33.1 \%$ reportaron haber recibido este tipo de medicamentos luego de su diagnóstico de enfermedad de Graves. ${ }^{(3)}$

Algunos pacientes hipertiroideos menos frecuentemente en la edad pediátrica se presentan con síntomas mentales atípicos; lo que se conoce como hipertiroidismo apático, en el cual el paciente se manifiesta con síntomas de depresión, apatía, somnolencia y pseudodemencia, en la ausencia de los síntomas y signos usuales de hipertiroidismo, pero en los cuales se demuestra la alteración bioquímica usual de las hormonas tiroideas. $^{(1)}$

La causa específica de la asociación entre psicosis $\mathrm{y}$ otros trastornos psiquiátricos y desórdenes tiroideos todavía se desconoce; aunque se han propuesto varias teorías. Marangell y colaboradores proponen que la hormona liberadora de tirotropina funciona como un neurotransmisor, con propiedades antidepresivas; y que usualmente está alterada en pacientes con hipertiroidismo. ${ }^{(4)}$ Por otra parte, Musselman y colaboradores proponen que los pacientes con enfermedad tiroidea primaria tienen altos índices de depresión y trastornos de comportamiento debido a alteraciones en el eje hipotálamo- hipófisis-adrenales y que estas consisten en cambios en la respuesta de la TSH a la TRH y concentraciones elevadas de TRH en el líquido cefaloraquídeo. ${ }^{(5)}$ Otros proponen una asociación causal entre la disminución de la 5hidroxitiramina cerebral y la aparición de síntomas depresivos en pacientes con trastornos tiroideos primarios. ${ }^{(6)}$

En cuanto al tratamiento, muchos endocrinólogos pediatras recomiendan las drogas antitiroideas como la primera línea de tratamiento, aunque otras terapias como la terapia con yodo radioactivo han venido ganando aceptación para niños mayores de 10 años y adolescentes como segunda línea de tratamiento. La tiroidectomía subtotal es un tratamiento seguro e igualmente efectivo. $^{(7)}$

En general se habla de que la mayoría de niños con enfermedad de Graves presentan adecuada respuesta al tratamiento, con casi un $87-100 \%$ de pacientes que llegan a estar en estado eutiroideo en unas pocas semanas a meses. ${ }^{(8)}$

En cuanto a la psicosis aguda; una vez que se sospecha que la causa es orgánica, el primer paso es diagnosticar y tratar la causa. El tratamiento adecuado requiere de una combinación de farmacoterapia y varias intervenciones psicosociales; esto dependerá de la fase clínica en la que se encuentre: en la fase prodrómica el niño o adolescente experimenta un período de deterioro funcional caracterizado por aislamiento social, preocupaciones y comportamientos idiosincráticos y dificultades académicas; en la fase aguda es cuando el paciente presenta alucinaciones visuales y/o auditivas y en la fase de recuperación; los síntomas se disipan. Existe una cuarta fase o residual en la cual los síntomas de alucinaciones no están presentes pero el niño se muestra apático, con falta de motivación y con afecto aplanado. ${ }^{(9,10)}$

La farmacoterapia se instituye en un intento para tratar la causa subyacente del cuadro psicótico. Generalmente, el tratamiento se inicia en niños ante la presencia de alucinaciones, sin embargo, no existe consenso sobre el uso de medicamentos antipsicóticos en la fase prodrómica en niños. ${ }^{(11)}$

Los agentes terapéuticos de primera línea incluyen los neurolépticos tradicionales que bloquean los receptores dopaminérgicos o los antipsicóticos atípicos; que poseen también efecto antagónico de 
los receptores de serotonina. Estos últimos suelen asociar menores efectos adversos, sin embargo, a excepción de la Clozapina, también se asocian a disquinesia tardía. ${ }^{(12,13)}$

En niños se han utilizado risperidona y olanzapina a partir de los 12 años de edad. Estos tienen menor efecto sedante que los neurolépticos tradicionales como el Haloperidol, Flufenazina, Tioridazina y Clorpromazina.

Se debe complementar el tratamiento farmacoterapéutico con intervenciones psicosociales de otras condiciones como el abuso de sustancias, depresión y tendencias suicidas. ${ }^{(12)}$

\section{CONCLUSIONES}

La enfermedad de Graves es una enfermedad autoinmune que constituye la principal causa de hipertiroidismo en la infancia y, aunque de manera ocasional, puede presentarse como un cuadro psicótico agudo.

Inicialmente, se deberá diferenciar si este es de origen orgánico o funcional, y posteriormente, tratar la causa subyacente.

No existe un consenso en niños para el uso de farmacoterapia en el pródromo de la psicosis asociada a hipertiroidismo.

Se debe complementar el tratamiento farmacológico con intervenciones psicosociales para identificar los factores de riesgo asociados y las condiciones del entorno donde vive el niño o adolescente.

\section{REFERENCIAS}

1. Bunevicius, R., Prange, ArthurJ. Psychiatric Manifestations of Graves'Hyperthyroidism:

Pathophysiology and Treatment Options. Therapy In Practice. CNS Drugs 2006; 20 (11), 897-909.

2. Weetman AP. Graves'disease. N Engl J Med 2000; 343: 1236-48.

3. Stern, Robert A., et al. A Survey Study of Neuropsychiatric Complaints in Patients with Graves' Disease. The Journal of Neuropsychiatry and Clinical Neurosciences. 1996; 8:181-185.

4. Marangell B., Callahan AM. Mood disorders and the thyroid axis. Curr
Opinion in Psychiatry 1998; 11: 67-70.

5. Musselman DL, Nemerott CB. Depression and endocrine disorders. Focus on the thyroid and adrenal system. Br J Psychiatry (Suppl) 1998; 30: 123128.

6. Cleare AK, McGergor A, O'Keane V. Neuroendocrine evidence for an association between hypothyroidism, reduced central 5-HT activity and depression. ClinEndocrinol 1995; 43: 713-719.

7. Rivkees SA, Sklar C, Freemark M. Clinical review 99: The management of Graves'disease in children, with special emphasis on radioiodine treatment. J ClinEndocrinolMetab 1998; 83;3767.

8. Rivkees SA, Szarfman A. Dissimilar hepatotoxicity profiles of propylthiouracil and methimazole in children. J ClinEndocrinolMetab 2010; 95;3260.

9. Pao M, Lohman C, Gracey D, et al. Visual, tactile, and phobic hallucinations: recognition and management in the emergency department. Pediatr Emerg Care. 2004;20:30-34.

10. Kanwae, Ajit, S. Sidhu. Hallucinations in children: Diagnostic and treatment strategies. Current Psychiatry Vol.9, No. 10, October 2010.

11. Varese, Fillipo, et al. Childhood Adversities Increase the Risk of Psychosis: A Meta-analysis of PatientControl, Prospective- and Cross-sectional Cohort Studies. Schizophrenia Bulletin Advance Access. March 29, 2012.

12. Read J, Bentall R, Dillon J, eds. Models of Madness: Psychological, Social and Biological Approaches to Psychosis. 2nd ed. London: Routledge. In press. 2006.

13. Barrio R, López-Capapé M, MartínezBadás I, et al. Graves' disease in children and adolescents: response to long-term treatment. ActaPaediatr 2005; 94:1583.

\section{CONFLICTO DE INTERES}

Los autores declaran que no existió ningún conflicto de interés en el presente reporte. 